\title{
Efectos dosis respuesta del ácido acetilsalicílico con cafeína en plantas de frijol (Phaseolus vulgaris) mediante metodología casera
}

\section{Effects doses responses of acetylsalicylic acid with caffeine in plants of kidney beans (Phaseolus vulgaris) through home methodology}

\author{
Karelys Barrios ${ }^{1}$, Dídimo Cruz ${ }^{1}$, Daniel Suárez $z^{1}$, Viccelda Domínguez ${ }^{2 *}$ \\ ${ }^{1}$ Licenciatura en Ingeniería Ambiental, Facultad de Ingeniería Civil, Universidad Tecnológica de Panamá \\ ${ }^{2}$ Departamento de Hidráulica, Sanitaria y Ciencias Ambientales, Facultad de Ingeniería Civil, Universidad Tecnológica de \\ Panamá
}

\begin{abstract}
Resumen El objetivo principal de este estudio es evaluar los efectos dosis respuesta del medicamento ácido acetilsalicílico con cafeína en plantas Phaseolus vulgaris, ejecutando una metodología casera; las concentraciones a las que fueron sometidas las plantas son $18.3 \mathrm{mg} / \mathrm{ml}, 36.7 \mathrm{mg} / \mathrm{ml}, 55 \mathrm{mg} / \mathrm{ml}$ y $73.3 \mathrm{mg} / \mathrm{ml}$ de ácido acetilsalicílico con cafeína; los ensayos se realizaron en triplicado. Como resultado se observó que, al graficar las medidas de altura, así como también los cambios en las hojas; a mayor concentración de medicamento $(73.3 \mathrm{mg} / \mathrm{ml}$ y $55 \mathrm{mg} / \mathrm{ml})$ se observa un daño más rápido y dramático en la estructura, coloración, salud y aspecto en general de la planta. Por otra parte, presentaron una media de diferencias de decrecimiento entre 5 y $6 \mathrm{~cm}$ por cada día, mientras que las plantas con las dos exposiciones más bajas pertenecientes a dosis de $36.7 \mathrm{mg} / \mathrm{ml} \mathrm{y} 18.3 \mathrm{mg} / \mathrm{ml}$ mostraron cambios de entre 2 y $4 \mathrm{~cm}$ por cada día de exposición al ácido acetilsalicílico con cafeína. Concluyendo, se pudo evaluar la diferencia en los efectos ocasionados por diferentes concentraciones de ácido acetilsalicílico con cafeína en la especie $P$. vulgaris, coincidiendo con otros estudios en los cuales a concentraciones menores $(2.1 \mathrm{mg} / \mathrm{mL})$ se encontraron efectos positivos del medicamento. Se recomienda realizar futuros estudios incluyendo análisis en laboratorio de suelo de $\mathrm{pH}$, porcentaje de finos, nutrientes, entre otros.
\end{abstract}

Palabras clave Ácido acetilsalicílico, cafeína, Phaseolus vulgaris, toxicología, tóxico.

\begin{abstract}
The main objective of this study is to evaluate the dose response effects of the drug acetylsalicylic acid with caffeine in Phaseolus vulgaris plants, using a homemade methodology; the concentrations to which the plants were subjected are $18.3 \mathrm{mg} / \mathrm{ml}$, $36.7 \mathrm{mg} / \mathrm{ml}, 55 \mathrm{mg} / \mathrm{ml}$ and $73.3 \mathrm{mg} / \mathrm{ml}$ of acetylsalicylic acid with caffeine; the tests were carried out in triplicate. As a result, it was observed that, when graphing the height measurements, as well as the changes in the leaves; the higher the drug concentration $(73.3 \mathrm{mg} / \mathrm{ml}$ and $55 \mathrm{mg} / \mathrm{ml}$ ), a more rapid and dramatic damage is observed in the structure, coloration, health and general appearance of the plant. On the other hand, they presented a mean difference of decrease between 5 and $6 \mathrm{~cm}$ for each day, while the plants with the two lowest exposures belonging to doses of $36.7 \mathrm{mg} / \mathrm{ml}$ and $18.3 \mathrm{mg} / \mathrm{ml}$ showed changes between 2 and $4 \mathrm{~cm}$ for each day of exposure to acetylsalicylic acid with caffeine. In conclusion, it was possible to evaluate the difference in the effects caused by different concentrations of acetylsalicylic acid with caffeine in the P. vulgaris species, coinciding with other studies in which positive effects of the drug were found at lower concentrations $(2.1 \mathrm{mg} / \mathrm{mL})$. It is recommended to carry out future studies including laboratory analysis of soil $\mathrm{pH}$, percentage of fines, nutrients, among others.
\end{abstract}

Keywords Acetylsalicylic acid, caffeine, Phaseolus vulgaris, toxicology, toxic.

*Corresponding author: viccelda.dominguez@utp.ac.pa

\section{Introducción}

El ácido acetilsalicílico (AAS) con cafeína (C), ha sido utilizado a lo largo de la historia demostrando ser uno de los fármacos más exitosos del mundo [1]. Principalmente es utilizado para el tratamiento del dolor aunado a su versatilidad lo hacen necesario para combatir nuevas enfermedades, además de poseer una buena tolerancia por el organismo. Por otra parte, cuenta con un amplio margen de seguridad y es de fácil manejo por el médico tratante [2].
Medicamentos cuyos ingredientes activos son el AASC han aliviado el dolor, la fiebre y la inflamación de millones de personas, desde hace ya más de un siglo [3]. Y es que este compuesto principal, el ácido acetilsalicílico, ha sido utilizado en proyectos de estudio con plantas donde se ha demostrado, por ejemplo, el incremento en la producción de granos por espigas de trigo [4]. También se ha demostrado que el ácido acetilsalicílico incrementa la altura de las plantas de haba en dosis de 1,5 mg por litro de agua, mejora la longitud y densidad de las raíces. Sin embargo, en esa investigación observaron que 
las plantas sufren una fitotoxicidad a dosis mucho mayores a la señalada [5].

Importantes departamentos como el de Agricultura de los Estados Unidos han descubierto que el ácido acetilsalicílico producía una mejor respuesta en las plantas aplicando una dosis recomendada de $100 \mathrm{mg} / \mathrm{L}$, para prepararlas mejor contra el ataque microbiano o de insectos [6]. Sin embargo, en esta investigación se tomó como base una concentración mayor, específicamente $500 \mathrm{mg} / \mathrm{L}$, dosis en la que se producen reacciones tóxicas en seres humanos [7].

Por su parte la cafeína es un alcaloide derivado de la purina, el cual se encuentra de forma natural. Existen alrededor de 100 especies vegetales entre las que se pueden mencionar hojas de té, nueces de cola, café y granos de cacao; también se puede encontrar en el chocolate, plantas de yerba mate y guaranás [8]. En su estado puro, la cafeína es un polvo blanco de sabor amargo. Su solubilidad a $25^{\circ} \mathrm{C}$ es de $21 \mathrm{mg} / \mathrm{ml}$, su DL50, es decir, la dosis que provoca la muerte en el $50 \%$ de los individuos es de $105 \mathrm{mg} / \mathrm{kg}$ en ratas [9]. Estudios han incidido en el papel de la cafeína como inductor de crecimiento vegetal e incluso como reductor del mismo, asociado a efectos alelopáticos tanto interespecífico como intraespecífico [10].

Se eligió la especie Phaseolus vulgaris principalmente debido a su rápido crecimiento y por ser una especie de consumo común en Centroamérica y Panamá. También se puede observar que esta especie ha sido utilizada en gran variedad de estudios, por ejemplo, para estimar el intervalo de concentración donde se encuentra el límite de tolerancia de $P$. vulgaris al mercurio (II) [11]. Otros resultados sobre el efecto de sustancias a las que han sido expuestas mostraron que esta especie es más sensible en las respuestas que otras especies como el maíz, especialmente a concentraciones altas [12].

No se han encontrado gran cantidad de estudios relacionados a los efectos dosis respuestas de AASC en $P$. vulgaris, lo cual es de importancia ya que se ha demostrado que los medicamentos pueden ser contaminantes emergentes de los diferentes ecosistemas [13]. Por lo tanto, este proyecto es una contribución al vacío del conocimiento en esta área y además un aporte metodológico como instrumento para cumplir con objetivos del desarrollo sostenible [14] como lo son garantizar la salud y bienestar (3), agua limpia y saneamiento (6), producción y consumo responsable (12) y vida de ecosistemas terrestre (15). El objetivo principal de este estudio es evaluar los efectos dosis respuesta del medicamento ácido acetilsalicílico con cafeína en plantas Phaseolus Vulgaris, poniendo en marcha una metodología casera.

\section{Metodología}

Para llevar a cabo este proyecto tomando en cuenta las limitaciones de movilidad por la pandemia mundial provocada por la COVID-19, se desarrolló una metodología replicable que se puede aplicar completamente desde casa como se presenta en la figura 1 .

\subsection{Recopilación de información}

Para empezar este proyecto primero se realizó la revisión bibliográfica donde se encontraron autores como Cano (2014) [5], Montes (2019) [10], en los que usaron el ácido acetilsalicílico y cafeína en Phaseolus Vulgaris, donde se pudo observar los efectos y otras características que se dan en los ensayos de toxicidad. A partir de la información encontrada teniéndola como base se procede a esquematizar y definir los parámetros y pasos a seguir para crear el diseño experimental.

\subsection{Diseño experimental}

El ensayo se estableció bajo condiciones de temperatura ambiente en cada una de las residencias de los 4 integrantes del grupo, siendo estas en el área de Panamá y Arraiján. Se realizaron 3 réplicas y un control con plantas de la especie $P$. vulgaris por cada integrante (Figura 1). Cada integrante aplicó a sus plantas una dosis diaria de AASC (ácido acetilsalicílico$\mathrm{C}_{9} \mathrm{H}_{8} \mathrm{O}_{4} 0.5 \mathrm{~g}$ y Cafeína-C8H10N4O2 $0.05 \mathrm{~g}$ ) diluida en $30 \mathrm{ml}$ de agua y $30 \mathrm{ml}$ de agua a la planta de control. Las dosis diarias aplicadas por cada integrante a cada planta fueron de $2.2 \mathrm{~g}$, $1.65 \mathrm{~g}, 1.1 \mathrm{~g}, 0.55 \mathrm{~g}$ respectivamente.

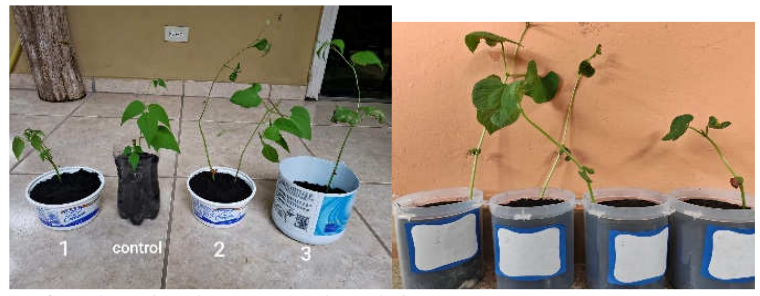

Figura 1. Colocación de muestras de cada integrante.

\subsection{Materiales}

Para la realización de este ensayo se utilizaron materiales de fácil acceso, los cuales son de uso común en las casas, estos fueron:

- P. vulgaris (Porotos rojos - Amapola)

- Tabletas del medicamento (AASC)

- Vasos desechables

- Envases plásticos de helado (de $946 \mathrm{ml}$ )

- Vasos graduados (en ml)

- Pesa de cocina (Maxihouse)

- Algodón

- Agua

- Regla y cinta de medir

- Tierra negra enriquecida (Melo)

- Dispositivo móvil

- Aplicación móvil para medir área foliar (Petiole)

- Aplicación móvil para medir temperatura (Tiempo)

- Aplicación móvil para medir coloración (Adobe Capture)

\subsection{Procedimiento}

En la figura 2 se observan imágenes relacionadas a los pasos del procedimiento. El procedimiento consistió en: 
1. Se escogió las semillas de porotos P. vulgaris de la misma marca (Amapola).

2. Se prepararon vasos con algodón para empezar la germinación de las semillas.

3. Se colocaron las semillas pegadas a las paredes de los envases debajo de la capa superficial de algodón.

4. Se estableció la cantidad de agua de $10 \mathrm{~mL}$ para aplicarle todos los días a la misma hora (5:30 pm) hasta empezar la dosificación del medicamento en las plantas.

5. Se verificó la temperatura cada día en la aplicación del celular a la misma hora de aplicarles el agua.

6. Después de 11 días de germinación, se decidió trasplantar a cada uno de los envases, ya que, las plantas tenían un tamaño adecuado para iniciar la dosificación.

7. Al contar con solo una pesa de cocina entre los integrantes, esa persona tomó un vaso graduado que llegaba hasta $300 \mathrm{~mL}$ que equivalía a un peso de 356.7 g. Por lo tanto, cada integrante, teniendo la información de densidad de $1.19 \mathrm{~g} / \mathrm{mL}$, colocó tierra en un vaso graduado hasta $300 \mathrm{~mL}$, lo que equivale a $356.7 \mathrm{~g}$ de peso. En triplicado.

8. Cada integrante repitió el paso de medir el volumen de tierra en los vasos graduados hasta completar los 900 ml y se le agregó a los envases que destinamos para trasplantar.

9. Se procedió a aplicar las dosis diarias del medicamento a las plantas, diluidas en $30 \mathrm{ml}$ de agua y a la planta control solamente $30 \mathrm{ml}$ de agua. El primer integrante agregó $2.2 \mathrm{~g}$ del medicamento a cada una de sus plantas, el segundo agregó $1.65 \mathrm{~g}$ a cada una de sus plantas, el siguiente $1.1 \mathrm{~g}$, y el cuarto integrante $0.55 \mathrm{~g}$, siendo estas dosis equivalentes a 4, 3, 2 y 1 tabletas del medicamento respectivamente.

10. Se tomaron apuntes a la misma hora $(5: 30 \mathrm{pm})$, todos los días, de los siguientes parámetros: altura, temperatura, humedad.

11. Después de aplicar las dosis diarias a la misma hora, también se observaron características físicas como: el color, estado de las hojas y el tallo.

12. Se hizo un cálculo del $\mathrm{pH}$ aproximado de las concentraciones utilizando la fórmula empírica del ácido acetilsalicílico y tomando en cuenta que es un ácido monoprótico débil de constante de acidez $\mathrm{Ka}=$ 2,64. $10-5 \mathrm{~mol} / \mathrm{L}$ [15].

13. Se dejó de aplicar el medicamento después de 8 días de haber iniciado la dosificación.

14. Se recopilaron, analizaron y graficaron los datos obtenidos de cada integrante.

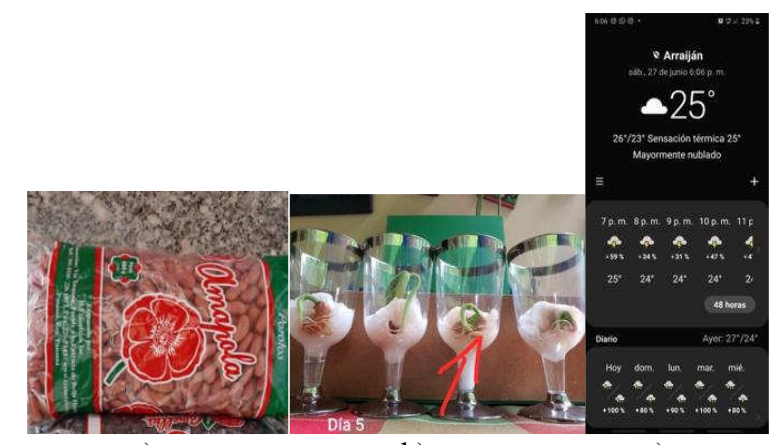

a)

b)

c)

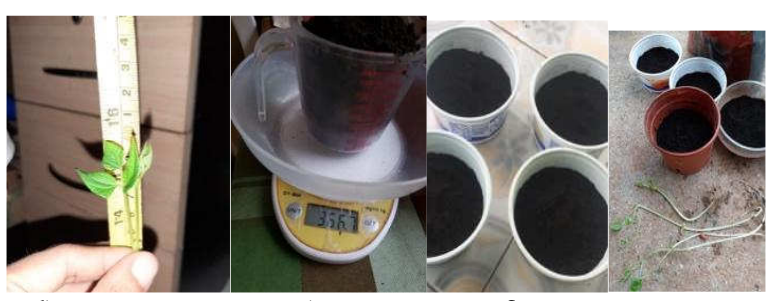

d)

e)

f)

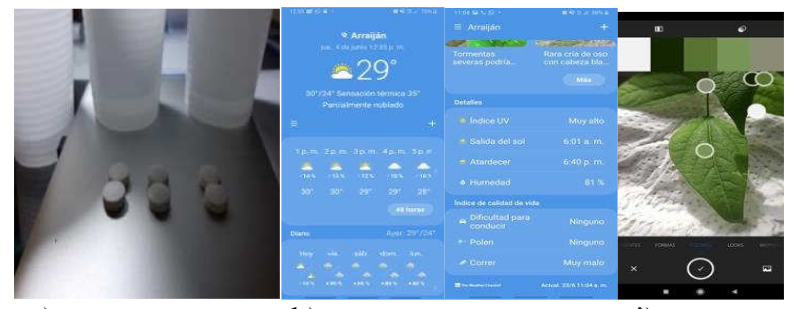

g)

h)

i)

Figura 2. Procedimiento para el desarrollo del ensayo: a) Selección de la semilla. b) Germinación de las semillas. c) Medición de factores ambientales durante el pre-ensayo. d) Medición del crecimiento de las plantas. e) Medición de peso y cantidad de tierra. f) Selección del envase y trasplante. g) Preparación de las dosis. h) Medición de parámetros ambientales durante el ensayo. i) Observación de cambios en las características físicas.

\section{Resultados y discusión}

De los ensayos realizados en casa por cada uno de los integrantes del grupo, se obtuvieron resultados según las dosis diarias aplicadas a las plantas, las cuales contenían diferentes cantidades de AAS y cafeína según la dosis correspondiente de cada integrante (figura 3 ).

Cada tableta de $550 \mathrm{mg}$ del medicamento (AASC) utilizado contiene $500 \mathrm{mg}$ de ácido acetilsalicílico, es decir, el $90.9 \%$ del contenido total, y 50 mg de cafeína correspondiente al $9.1 \%$ restante. 


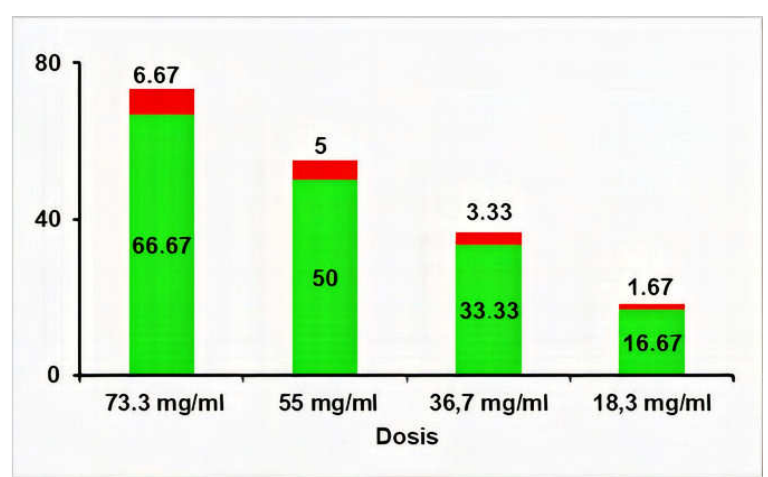

Figura 3. Cantidades de ácido acetilsalicílico (ఏ) y cafeína (ఏ) en las distintas dosis.

\subsection{Efectos generales del ácido acetilsalicílico y de la cafeína en las plantas}

En las doce plantas expuestas al medicamento, se analizó el tamaño y la coloración de estas como las características de respuesta del organismo a la aplicación del químico (tabla 1). En cada una de ellas se evidenció una notable reducción de su tasa de crecimiento, alteración en sus tallos, hojas y coloración. A diferencia de los resultados de Cano (2014) que propone un efecto positivo del ácido sobre las plantas [5]. Como era de esperarse, se hace mucho más evidente las variaciones en la salud de las plantas al compararlas 120 horas desde la primera dosis colocada el primer día del ensayo, ya que se sobrepasó la dosis recomendada en otros ensayos por autores como Suszkiw del Departamento de Agricultura de Estados Unidos [6].

Tabla 1. Características generales de las plantas de frijol (Phaseolus vulgaris) el primer día de aplicación de las dosis de ácido acetilsalicílico y cafeína

\begin{tabular}{|c|c|c|}
\hline Dosis & $\begin{array}{l}\text { Aspecto del } \\
\text { tallo }\end{array}$ & $\begin{array}{l}\text { Características } \\
\text { de las hojas }\end{array}$ \\
\hline$(73.3 \mathrm{mg} / \mathrm{ml})$ & $\begin{array}{l}\text { Verde claro } \\
\text { y saludable }\end{array}$ & $\begin{array}{l}\text { Buen estado y } \\
\text { gran tamaño }\end{array}$ \\
\hline$(55 \mathrm{mg} / \mathrm{ml})$ & $\begin{array}{c}\text { Verde } \\
\text { oscuro y } \\
\text { saludable }\end{array}$ & $\begin{array}{l}\text { Verdes y } \\
\text { saludables }\end{array}$ \\
\hline$(36.7 \mathrm{mg} / \mathrm{ml})$ & $\begin{array}{l}\text { Verde claro } \\
\text { y saludable }\end{array}$ & $\begin{array}{c}\text { Pequeñas y } \\
\text { saludables }\end{array}$ \\
\hline$(18.3 \mathrm{mg} / \mathrm{ml})$ & $\begin{array}{c}\text { Completam } \\
\text { ente } \\
\text { saludables }\end{array}$ & $\begin{array}{c}\text { Pequeñas, verdes } \\
\text { y saludables }\end{array}$ \\
\hline
\end{tabular}

La característica física más notable fue el cambio de coloración en las plantas (tabla 2). Si consideramos que el medicamento utilizado es un ácido débil y que a medida que se aplicaron las dosis diarias en concentraciones con $\mathrm{pH}$ que variaron entre los 2.5 a 2.8 se puede decir que el medio en el que fueron aplicadas pudo disminuir significativamente su $\mathrm{pH}$ [16]. Un efecto que concuerda Rivera (2018) quien observó que las plantas expuestas a sustancias ácidas o niveles de $\mathrm{pH}$ bajos presentan en general un aspecto raquítico y amarillento, las hojas se ven más claras que el color verde pálido, y van tornándose en amarillo hasta llegar a colores más oscuros [17].

Tabla 2. Características generales de las plantas de frijol (Phaseolus vulgaris) en el quinto día luego de ser aplicada las dosis de ácido

\begin{tabular}{|c|l|l|}
\hline \multicolumn{1}{|c|}{ Dosis } & $\begin{array}{c}\text { Aspecto del } \\
\text { tallo }\end{array}$ & $\begin{array}{l}\text { acetilsalicílico y cafeína (día 9) } \\
\text { Características } \\
\text { de las hojas }\end{array}$ \\
\hline \multirow{3}{*}{$(73.3 \mathrm{mg} / \mathrm{ml})$} & $\begin{array}{l}\text { Dobladas y } \\
\text { de color } \\
\text { verde oliva }\end{array}$ & $\begin{array}{l}\text { Muy poca vida } \\
\text { y deteriorándose } \\
\text { casi en su } \\
\text { totalidad }\end{array}$ \\
\hline$(55 \mathrm{mg} / \mathrm{ml})$ & $\begin{array}{l}\text { Caído en su } \\
\text { totalidad }\end{array}$ & $\begin{array}{l}\text { Secas e inertes } \\
\text { completamente }\end{array}$ \\
\hline \multirow{3}{*}{$\begin{array}{l}\text { A punto de } \\
\text { caer y con } \\
\text { quemaduras }\end{array}$} & $\begin{array}{l}\text { Hojas con poca } \\
\text { vitalidad y con } \\
\text { distintos tonos }\end{array}$ \\
\hline$(18.3 \mathrm{mg} / \mathrm{ml})$ & $\begin{array}{l}\text { Dificultades } \\
\text { en } \\
\text { mantenerse } \\
\text { por sí sola }\end{array}$ & $\begin{array}{l}\text { Hojas inferiores } \\
\text { marchitas casi } \\
\text { por completo }\end{array}$ \\
\hline
\end{tabular}

\subsubsection{Efecto en el crecimiento y desarrollo de las plantas}

El día 22 de junio se inició la exposición de las doce plantas al medicamento (AASC), los resultados registrados en los días posteriores mostraron evidencias significativas del efecto del ácido acetilsalicílico con la cafeína, mostrando un notable deterioro en aquellas plantas donde las concentraciones del fármaco eran más altas. A diferencia de las plantas expuestas a concentraciones más bajas que mostraron efectos negativos un poco más lentos y pausados. Muestra de esto es que las plantas con dosis de $55 \mathrm{mg} / \mathrm{mL}$ presentaban daños notorios en sus hojas superiores en menos de 24 horas luego de aplicado el medicamento (AASC), en las plantas expuestas a las dosis de $18.3 \mathrm{mg} / \mathrm{ml}$ su tiempo de reacción a los efectos del medicamento fueron más tardías, como se representa en más detalladamente en la figura 4, donde se puede observar los días en los que tuvo un efecto negativo en la vida de las plantas.

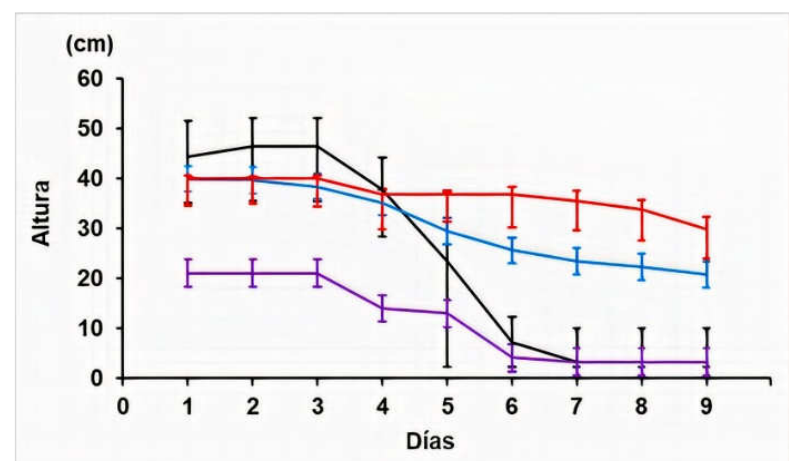

Figura 4. Cotejo del decaimiento al triplicado de las plantas provocado por la exposición a distintas dosis, (\36.7 mg/ml), ( $\square 3.3 \mathrm{mg} / \mathrm{ml}),(\square 18.3 \mathrm{mg} / \mathrm{ml})$, $55 \mathrm{mg} / \mathrm{ml})$. 
Como queda demostrado al graficar las medidas de altura en la figura 4, como también los demás cambios morfológicos por las dos plantas con mayor concentración de medicamento ( $73.3 \mathrm{mg} / \mathrm{ml}$ y $55 \mathrm{mg} / \mathrm{ml})$ se observa un daño más rápido y dramático a la estructura, coloración, salud y aspecto en general de la planta. A su vez las dos plantas con menor concentración $(36.7 \mathrm{mg} / \mathrm{ml}$ y $18.3 \mathrm{mg} / \mathrm{ml})$, al ser su altura graficada presentan una curva de decrecimiento más suave y paulatino. Las plantas con dosificación de $73.3 \mathrm{mg} / \mathrm{ml}$ y 55 $\mathrm{mg} / \mathrm{ml}$, presentaron una media de diferencias de decrecimiento entre 5 y $6 \mathrm{~cm}$ por cada día, mientras que las plantas con las dos exposiciones más bajas pertenecientes a dosis de 36.7 $\mathrm{mg} / \mathrm{ml}$ y $18.3 \mathrm{mg} / \mathrm{ml}$ mostraron cambios de entre 2 y $4 \mathrm{~cm}$ por cada día de exposición al AASC.

Para una visión más simplificada se comparó el decrecimiento de los valores medios de las plantas con las distintas dosis y el crecimiento sin ningún tipo de dosis de la planta de control, dando como resultado la figura 5.

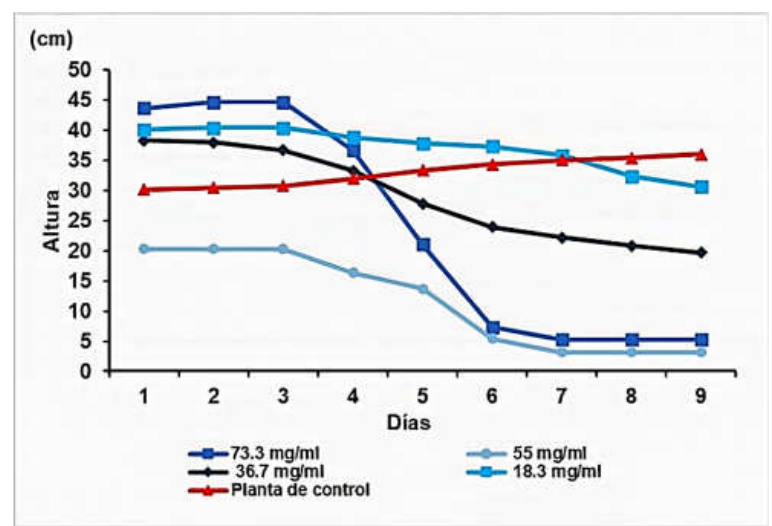

Figura 5. Comparación del promedio de las plantas de control con el promedio de las dosificadas con AASC.

En la figura 6 se muestra un modelo preliminar de la cinética de decaimiento por parte de la planta $\boldsymbol{P}$. vulgaris, en función de las pendientes y dosis suministradas del fármaco compuesto de AASC durante el experimento realizado.

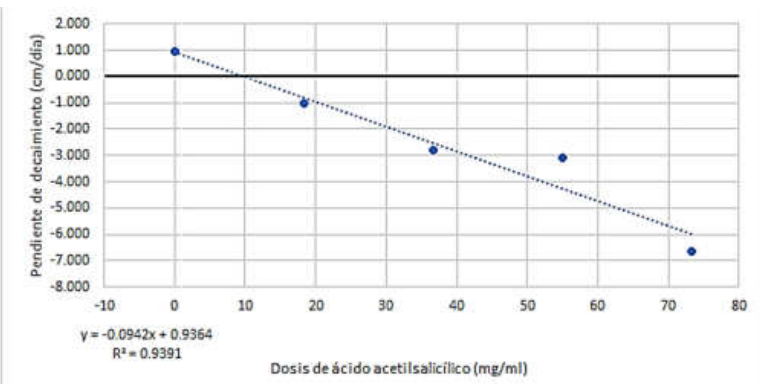

Figura 6. Modelo preliminar cinético de decaimiento de plantas de frijol Phaseolus vulgaris en función de la exposición a diferentes dosis de ácido acetilsalicílico.

\subsubsection{Análisis Estadístico. ANOVA}

Se realizó un análisis de varianza entre las variables como el decrecimiento por día causado a las plantas con las distintas dosis, los resultados mostraron diferencias entre ellas, analizándolas tanto individualmente como conjuntas. Como se observa en las $\mathrm{P}$ valor de la siguiente tabla 3.

Tabla 3. ANOVA obtenido de los experimentos
\begin{tabular}{|l|c|l|l|l|l|}
\hline Altura Dosis*Dia + error & & \\
\hline Df & Sum & Sq Mean & Sq F & value & Pr(>F) \\
\hline Dosis & 1 & 2207 & 2207 & 15.53 & $9.68 \mathrm{e}-05^{* * *}$ \\
\hline Dia & 1 & 13279 & 13279 & 93.44 & $<2 \mathrm{e}-16^{* * *}$ \\
\hline Dosis:Dia & 1 & 3632 & 3632 & 25.55 & $6.71 \mathrm{e}-07^{* * *}$ \\
\hline Residuals & 380 & 54007 & 142 & & \\
\hline
\end{tabular}

$\mathrm{Al}$ obtener diferencias en los resultados, posteriormente se realizó un análisis apareado entre las diferentes dosis para encontrar diferencias entre ellas (tabla 4).

\begin{tabular}{|l|l|l|}
\begin{tabular}{|l|l|l|}
\hline Pares de Dosis & Día 1 & Día 9 \\
\hline 0 vs $18.3 \mathrm{mg} / \mathrm{mL}$ & 0.435 & 0.03091 \\
\hline 0 vs $36.7 \mathrm{mg} / \mathrm{mL}$ & 0.533 & 0.00413 \\
\hline 0 vs $55.5 \mathrm{mg} / \mathrm{mL}$ & 0.435 & $1.4 \mathrm{e}-05$ \\
\hline 0 vs $73.3 \mathrm{mg} / \mathrm{mL}$ & 0.147 & $2.4 \mathrm{e}-05$ \\
\hline 18.3 vs $36.7 \mathrm{mg} / \mathrm{mL}$ & 1.000 & 0.04073 \\
\hline 18.3 vs $55.5 \mathrm{mg} / \mathrm{mL}$ & 0.322 & 0.00011 \\
\hline 18.3 vs $73.3 \mathrm{mg} / \mathrm{mL}$ & 1.000 & 0.00021 \\
\hline 36.7 vs $55.5 \mathrm{mg} / \mathrm{mL}$ & & 0.00508 \\
\hline 36.7 vs $73.3 \mathrm{mg} / \mathrm{mL}$ & 1.000 & \\
\hline 55.5 vs $73.3 \mathrm{mg} / \mathrm{mL}$ & 0.111 & 0.58365 \\
\hline
\end{tabular}
\end{tabular}

Haciendo la comparación del primer día con el último día se evidenció las diferencias entre las dosis con la planta de control y de igual forma hubo un cambio entre dosis excluyendo las dosis más altas $(73 \mathrm{mg} / \mathrm{mL}$ y $55 \mathrm{mg} / \mathrm{mL})$. Estas dosis más altas no mostraron mayor desigualdad, ya que los efectos que causaron sobre sus respectivas plantas en su gran mayoría no mostraron gran similitud en su tasa de decaimiento. 


\subsection{2 Áreas de las hojas}

El área de la hoja es una de las características físicas fundamentales para interpretar el comportamiento de la planta, y su asimilación a las distintas dosis de AAS con cafeína, sin embargo, la aplicación usada para medir el área foliar de las hojas necesita que la hoja sea arrancada en su totalidad de la planta lo que impediría su medición periódica tras ser expuesta a las distintas dosis. A pesar de ello se observó que a medida que transcurrían los días de exposición al medicamento (AASC) se notaron cambios radicales en las estructuras de las hojas, como en este caso su tamaño, el daño producido pueden ser observados en la figura 7, donde además del color y textura el área de las hojas sufre un cambio bastante marcado.

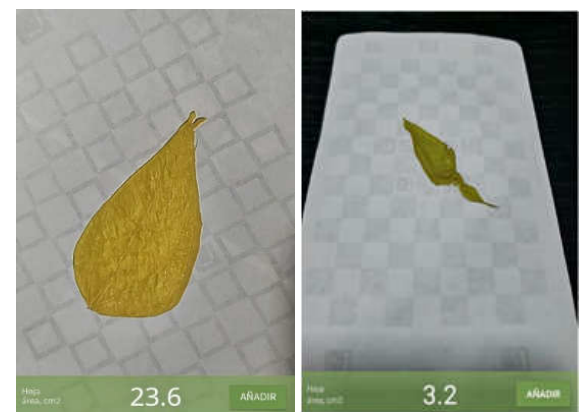

Figura 7. Aspectos de las hojas de Phaseolus Vulgaris afectada por el AASC

\subsection{Plantas de control}

Las plantas de control, sembradas, regadas y cuidadas bajo las mismas condiciones dentro de lo posible mostraron un crecimiento a distintos ritmos, sin embargo, siempre de forma exponencial como se muestra en la figura 8 .

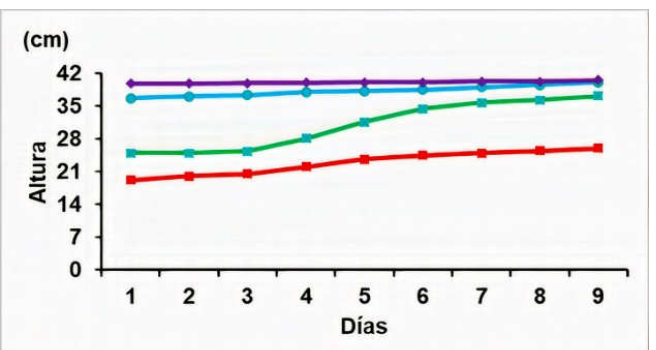

Figura 8. Crecimiento de las plantas de frijol $P$. Vulgaris usadas como control por cada integrante.

En esta figura se demuestra el crecimiento de cada planta de forma ascendente a lo largo de los días. Las diferencias en el crecimiento de las plantas se les atribuye a los factores ambientales de las distintas áreas donde se desarrollaron los ensayos [18].

En la semana antes de la exposición del AASC a las plantas se registró una diferencia promedio de dos grados de temperatura como se puede observar en la figura 9, donde se representa el crecimiento de las plantas respecto a la temperatura diaria medida a las 5:30 pm. Además, hubo un promedio de $10 \%$ de diferencia en la humedad, entre las áreas de Panamá y Panamá Oeste, lo que pudo afectar significativamente el desarrollo de las plantas.

a)

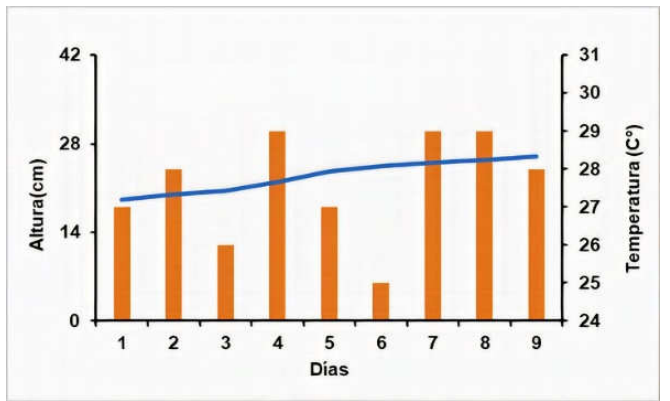

b)

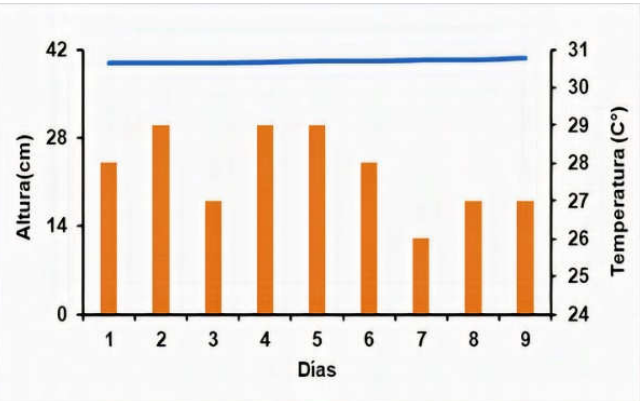

c)

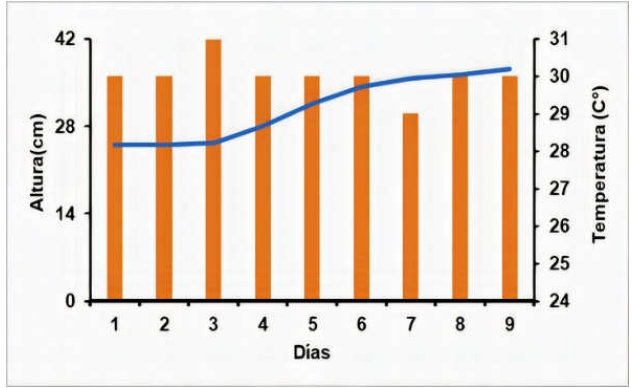

d)

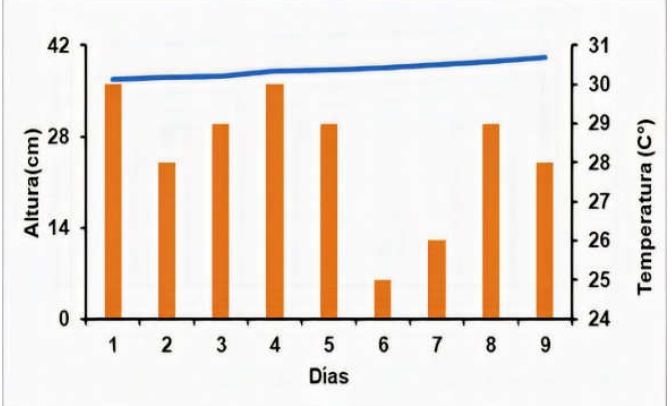

Figura 9. Crecimiento de la planta de frijol Phaseolus vulgaris usadas como planta control en concentraciones (a) $18.3 \mathrm{mg} / \mathrm{L}$, (b) $36.7 \mathrm{mg} / \mathrm{L}$, (c) $55 \mathrm{mg} / \mathrm{L}$ (d) $76.3 \mathrm{mg} / \mathrm{L}$, con respecto a la temperatura diaria 


\section{Conclusiones y recomendaciones}

- Se logró elaborar una metodología casera para desarrollar un ensayo dosis-respuesta que permitió observar los efectos tóxicos del medicamento en concentraciones iguales o mayores de $18.3 \mathrm{mg} / \mathrm{mL}$.

- Se pudo comparar la diferencia en los efectos ocasionados por el AASC a diferentes concentraciones en plantas de la especie $P$. vulgaris, y a su vez se pudo comparar con los resultados obtenidos por Cano (2014), coincidiendo con los efectos positivos del medicamento sobre las plantas a concentraciones menores (2.1 $\mathrm{mg} / \mathrm{mL}$ ) de las utilizadas en los ensayos de este documento.

- A mayor concentración de medicamento $(73.3 \mathrm{mg} / \mathrm{ml}$ y $55 \mathrm{mg} / \mathrm{ml}$ ) se observa un daño más rápido y dramático en la estructura, coloración, salud y aspecto en general de la planta. Por otra parte, presentaron una media de diferencias de decrecimiento entre 5 y $6 \mathrm{~cm}$ por cada día, mientras que las plantas con las dos exposiciones más bajas pertenecientes a dosis de $36.7 \mathrm{mg} / \mathrm{ml}$ y 18.3 $\mathrm{mg} / \mathrm{ml}$ mostraron cambios de entre 2 y $4 \mathrm{~cm}$ por cada día de exposición al ácido acetilsalicílico con cafeína.

- Las plantas de la especie $P$. vulgaris, además de ser plantas de rápido crecimiento también muestran rápidas reacciones a los efectos del medicamento (AASC), lo que permitió observar los efectos en pocos días.

- Se recomienda realizar más pruebas de las necesitadas por si algunas semillas no germinan, o si durante el proceso surge algo inesperado.

- Realizar futuros estudios incluyendo análisis en laboratorio de $\mathrm{pH}$, de suelo, porcentaje de finos, nutrientes, entre otros.

- Para encontrar el área de las hojas existen otros métodos en los cuales no son necesarios arrancar las hojas de las plantas, como utilizar una hoja milimétrica el cual no es un método del todo preciso, pero cuida de mejor manera la integridad de la planta.

\section{AGRADECIMIENTO}

Agradecemos a nuestro compañero de carrera, el Mgtr. Víctor Bravo por todo su apoyo en la parte estadística del proyecto.

\section{REFERENCIAS}

[1] M. F. Braña, L. A. Del Rio, C. Trives, y N. Salazar, "La verdadera historia de la Aspirina", Anales de la Real Academia Nacional de Farmacia, 2005. [En línea]. Disponible en: http://hdl.handle.net/10637/878.

[2] Félix A. Ramírez Labrada, D. A. (1999). La aspirina. El medicamento del siglo. Archivo Médico de Camagüey.

[3] G. V. Bibiana, "Aspirina: 120 años del primer medicamento "milagro", OpenMind BBVA, abril 2019. https://www.bbvaopenmind.com/ciencia/investigacion/aspirina -120-anos-del-primer-medicamento-milagro/
[4] R. L. Tejeda, V. Camacho Rodríguez, y M. A. Gutiérrez Coronado, "Aplicación de ácido salicílico para incrementar el rendimiento agronómico en tres variedades de trigo", Terra Latinoamericana, vol. 16, n.o 1, pp. 43-48, mar. 1998.

[5] N. D. Lucero Cano, "Determinación del efecto del elicitor ácido acetilsalicílico sobre el control de mancha chocolate (Botrytis fabae L.), en el cultivo de haba (Vicia faba L.)", Universidad Politécnica Estatal Del Carchi, Ecuador, 2014.

[6] S. Jan, "Priming Plant Defenses with Aspirin-like Compound", Agricultural Research Service U.S. Department of Agriculture, feb. 13, 2014. https://www.ars.usda.gov/newsevents/news/research-news/2014/priming-plant-defenses-withaspirin-like-compound/

[7] G. Fiorenza Bianzuzzi, D. Gonzalez, A. Perez, A. Ridolfi, y A. Strobl, "Manual de procedimientos analíticos toxicológicos para laboratorios de baja complejidad", Argentina, 2007. [En línea]. Disponible en: https://docplayer.es/1042090-Manual-deprocedimientos-analiticos-toxicologicos-para-laboratorios-debaja-complejidad.html en: http://www.msal.gob.ar/images/stories/ministerio/intoxicacion es/laboratorios/manual-procedimientos-analiticos.pdf

[8] A. Vázquez-Ovando, I. Ovando-Medina, L. Adriano-Anaya, D. Betancur-Ancona, y M. Salvador-Figueroa, "Alcaloides y polifenoles del cacao, mecanismos que regulan su biosíntesis y sus implicaciones en el sabor y aroma", Archivos latinoamericanos de nutrición, Vol. 66, no. 3, pp. 239-254, 2016. [En línea]. Disponible en: https://www.alanrevista.org/ediciones/2016/3/art-10/\#

[9] F. Camacho Ferre, Técnicas de producción en cultivos protegido. España, 2003. [En línea]. Disponible en: https://dialnet.unirioja.es/servlet/libro? codigo $=4827$

[10] O. Montes, H. Bojórquez, F. Toresano, F. Diánez, y F. Camacho, "Evaluación agronómica de la aplicación de cafeína en cultivo de pimiento bajo abrigo en el sureste de España", Actas de Horticultura, no. 60, p. 6, 2014.

[11] E. Sánchez, R. González, "Modelado básico de una metodología para la determinación y extracción de mercurio en muestras simuladas de fitorremediación con Phaseolus vulgaris", Revista Cubana de Química, vol. 23, no. 2, pp. 54-57, 2011

[12] Guzmán, G. I. "Efecto del plomo sobre la imbibición, germinación y crecimiento de Phaseolus vulgaris L. y Zea mays", L. Biotecnología Vegetal. Vol. 13, no. 3, 2013, mayo 2013. [En línea]. Disponible en:

https://revista.ibp.co.cu/index.php/BV/article/view/114.

[13] Reinoso, J., Serrano, C., Orellana, D. "Contaminantes emergentes y su impacto en la salud". Revista de la Facultad de Ciencias y Medicina. Universidad de Cuenca., mayo 2017.

[14] Organización de las Naciones Unidas, "Objetivos de Desarrollo Sostenible", 25 septiembre 2015. [En línea]. Disponible en: https://www.un.org/sustainabledevelopment/es/2015/09/laasamblea-general-adopta-la-agenda-2030-para-el-desarrollosostenible/.

[15] Reacciones de transferencia de protones (ácidos y bases). http://www.escritoscientificos.es/problemasfyq/problemasqui mica/06quiprob01a10/06quiprob05.htm

[16] R. Martínez. "Titulación ácido/base: determinación de aspirina en tabletas comerciales". Universidad de Puerto Rico Humacao. 2016.

https://www.cimat.mx/ciencia_para_jovenes/tcj/2016/material _curso_quimica/titulacion_aspirina.pdf 
Domínguez (et al): Efectos dosis respuesta del ácido acetilsalicílico con cafeína en plantas de frijol (Phaseolus vulgaris) mediante metodología casera

[17] E. Rivera, M. Sánchez, \& H. Domínguez. "pH como factor de crecimiento en plantas". Revista de Iniciación Científica, 4, 101-105. 2018. Disponible en: https://doi.org/10.33412/revric.v4.0.1829
[18] CANNA Research. "Influencia de la temperatura ambiental en las plantas". [En línea]. Disponible en:

http://www.canna.es/influencia_temperatura_ambiental_en_las _plantas 